\title{
A New Miniaturised Multi-Axis Force/Torque Sensors based on Optoelectronic Technology and Simply-Supported Beam
}

\author{
Yohan Noh, Joao Bimbo, Agostino Stilli, Helge Wurdemann, Member, IEEE, Hongbin Liu, Member, IEEE, \\ James Housden, Kawal Rhode, and Kaspar Althoefer, Member, IEEE
}

\begin{abstract}
This paper presents a methodology for the development of a multi-axis force/torque sensor based on optoelectronic technology. The advantages of using this sensing principle are the low manufacturing costs, the simple fabrication, and the immunity to electrical noise. The force/ torque sensor makes use of six optical sensors: each sensor measures the displacement of a reflective surface that moves integrally with a simply-supported beam. The proposed mechanical structure allows for a variety of shapes on the mechanical structure to be easily adaptable to many robot applications. In this paper, we present a five-axis force/torque sensor based on this optoelectronic principle. To measure force/torque components, two identical three-DoF force/torque sensor structures (comprised of three beams) are mounted on top of each other. Photo sensors and mirrors are fixed inside the structure to measure the six beam deflections. In this paper, we describe the sensor structure, design, fabrication, calibration, and verify our sensor development methodology.
\end{abstract}

\section{INTRODUCTION}

The advent of Robot Technology (RT) has assisted in manufacturing products in industry [1] and assist people at the help desks of convenient services, restaurants, cafes, etc. [2]. They also nurse patients in hospitals, as well as assist doctors carrying out surgical operations and other medical applications [3-4].

Presently, robots are closely living with people, and in the near future, we expect robots to be an everyday presence in our society. For this reason, scientists have studied how robots feel and express our five senses (sound, sight, touch, smell and taste) using various sensors such as tactile, pressure, force, gas sensor etc., so that robots can coexist and interact with humans [5-6]. Particularly, when using force sensors, robots can acquire the sense of touch, i.e. robots can recognize object shapes, distinguishing various kinds of tactile information such as pressure, scratch, prick, rub, and etc. [7-8]. For these reasons, the sense of touch has been extensively researched within the field of the robotics. For instance, robot hands can use tactile information to recognize an object's shape and grasp it with a certain force in order not to break it nor let it slip [9-10]. Force or torque control are

The research leading to these results has received funding from the Wellcome Trust IEH Award [102431] and King's College London Informatics Research Impact funding 2016.

Yohan Noh, Joao Bimbo, Hongbin Liu, and Kaspar Althoefer are with the Department of Informatics, King's College London, UK (e-mail corresponding author: yohan.noh@kcl.ac.uk).

Helge Wurdemann is with the Department of Mechanical Engineering, University College London, UK.

James Housden, and Kawal Rhode are with the Department of Biomedical Engineering, Division of Imaging Sciences and Biomedical Engineering, King's College London, UK

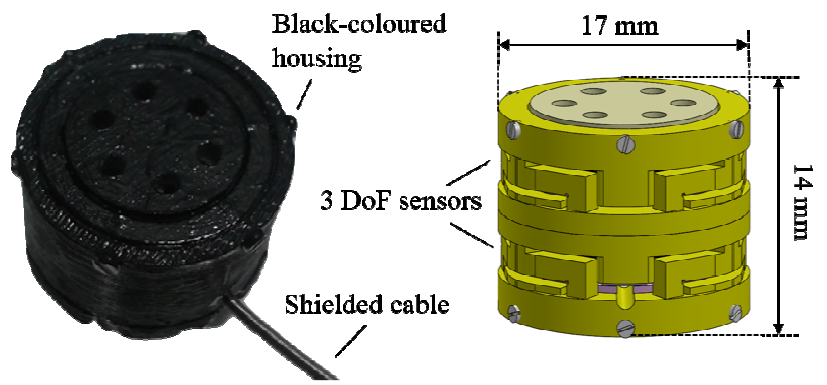

Figure 1: Prototype (left) and CAD drawing of the Five-axis force/torque sensor (right).

implemented into robotic nurse assistants not to apply excessive external force on the patients for safety [11-12]. In the field of industrial robotics, force and torque control are integrated to simulate stiffness on each of the robot joints, so they do not apply excessive external force to the assembly part. Further, force and torque control can be used by a robot to insert a tube into a pin, as it can make the robot feel its way into that assembly, providing flexibility in the manufacturing process [13].

A variety of force/torque sensors based on piezoresistive materials [14], strain gauges [15], polyvinylidene fluoride (PVDF) films [16], and fibre-optics [17], [18] have so far been proposed in the literature. A low-cost force/torque sensing principle which is also simple to fabricate makes use of optoelectronic technology. In [19-20], single-axis force and torque sensors were proposed and integrated into robots. In [21-22], tension sensors were integrated into tendon mechanisms for force control. A multi-axis force/torque sensor was proposed by [23-24] for applying stiffness control in each of the joints, and also integrated itself in the structure which provides ample space in the centre to facilitate cables and pipes through the sensor structure. The advantages of the use of optoelectronics are its immunity to electrical noise, low power consumption, low-level noise, no need for any electronic filtering, and low cost [22]. In addition, cutting edge technology allows photo sensors to be miniaturised. Recent advances have produced diminutive sized photo sensors such as the NJL5901R-2, $1.0 \times 1.6 \times 0.6 \mathrm{~mm}^{3}$, New Japan Radio Co., Ltd, Japan. Hence, the overall size of the sensor structure can also be reduced and miniaturised.

Here, we propose and investigate a new five-axis force/torque sensor based on the optoelectronic sensing principle (see Figure 1). The work presented here follows our overall idea of creating small low-cost, adaptable sensors that can be easily integrated into a variety of robotic manipulator with only minimal re-design efforts. In our solution, the sensor structure is inspired by our previous work presented in [17] [24] and is made of two three-axis sensors which are assembled in series. These sensors consist 
of a number of simply-supported beams that deflect when external forces are exerted. The deflection is measured by optical sensor. The presented mathematical model shows that five force and moment values $\left(\mathrm{F}_{\mathrm{x}}, \mathrm{F}_{\mathrm{y}}, \mathrm{F}_{\mathrm{z}}, \mathrm{M}_{\mathrm{x}}\right.$, and $\left.\mathrm{M}_{\mathrm{y}}\right)$ can be calculated considering the different amount of the deflections. Today's 3D printing technologies allow fabricating the sensing structure in ABS plastic and metal material at low cost. Due to the availability of diminutive sized photo sensors, we were able to miniaturise the proposed sensor, so that its dimensions are comparable to the commercially available ATI Nano 17 force/torque sensor.

The paper is organised as follows: In Section II, a list of requirements is identified that should be considered during the design process. Further, the mechanical design of the sensor structure and a FEM analysis is presented. The mathematical model of the beam structure is described. Using a modified linear motorised rail and an ATI Nano 17 force/torque sensor, the multi-axis force/torque sensor based on optoelectronics is calibrated (see Section III). The validation is presented in Section IV followed by the conclusions in Section V.

\section{Design Methodology AND FABRICATION}

\section{A. Design inputs}

The design inputs for the creation of this new force/torque sensor have been defined in order to go beyond what we achieved in our previously work presented [17] [24]. The following requirements should be satisfied:

- The new sensor should be capable of measuring multiaxis (more than three Degrees of Freedom (DoFs)) force/torque components. The desirable maximum measurements should include force values of up to $2 \mathrm{~N}$ and moments of up to $3 \mathrm{Ncm}$.

- The overall structural dimensions should be comparable to commercially available sensors such as the ATI Nano 17 force/torque sensor.

- Inspired by the mechanical structure in [17] [24], the fabrication of the new sensor structure should be at low cost.

- The mechanical design should be adaptable to a variety of robotic structures for effortless integration.

These performance indicators are considered in the design process in order to evaluate the design outputs.

\section{B. Mechanical design of the five-DoF force/torque sensor}

Inspired by the three-DoF force/torque sensors with a cantilever beam structure as described in [17] [24], the overall structure of this new sensor is made of two three-axis force/torque sensors based on simply-supported beam structures (see Figure 1 and Figure 2). This results in a sensing structure that is able to measure five force/torque components in total: $F_{x}, F_{y}, F_{z}, M_{x}$, and $M_{y}$. In this section, the mechanical design and sensing technique are described in detail. The sensing principle is based on optical sensors measuring the displacement of deflection caused by external forces or moments (Figure 3). The deformation, in turn, can be related to force/torque components. The structure of the proposed five-axis force/torque sensor is shown in and Figure 4. A flexible ring-like structure is fabricated of polymer ABS (Acrylonitrile Butadiene Styrene) by a rapid prototyping

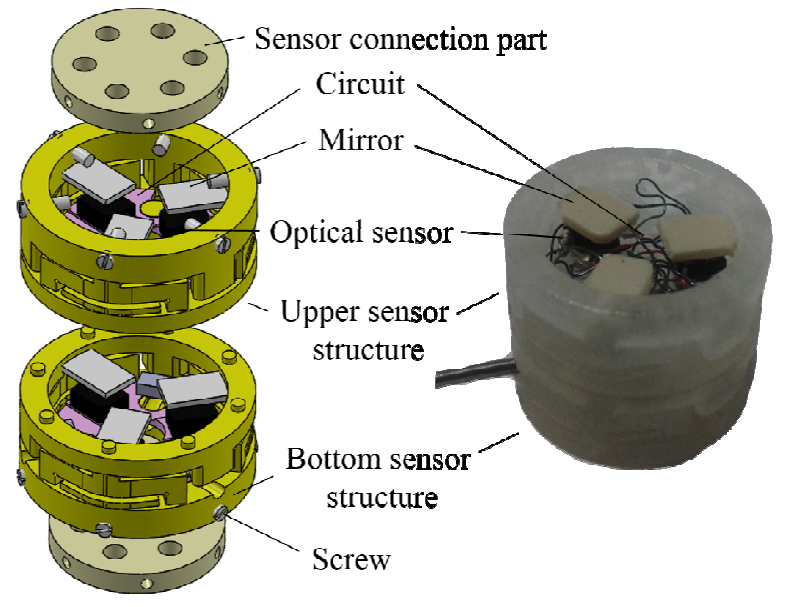

(a)

(b)

Figure 2: Assembly of the five-axis force/torque sensor: (a) Exploded view and (b) assembled prototype of the five axis force/torque sensor.

machine (Project HD-3000 Plus, 3D Systems). As mentioned earlier, two three-DoF sensing structures are attached on top of each other. Each element has three flexures (simplysupported beams) as illustrated in Figure 3 and Figure 4. Consequently, the overall sensor structure has six deflections $\left(\delta_{1}, \delta_{2}, \delta_{3}, \delta_{4}, \delta_{5}\right.$, and $\left.\delta_{6}\right)$ which can be measured and are the input to a calibration matrix to estimate $\mathrm{F}_{\mathrm{x}}, \mathrm{F}_{\mathrm{y}}, \mathrm{F}_{\mathrm{z}}, \mathrm{M}_{\mathrm{x}}$, and $M_{y}$. It is not possible to measure $M_{z}$ since the sensor structure constrains deformation with respect to rotational torque along the z-axis.

We were able to miniaturise the diameter of the five-DoF sensor by over $50 \%$ to $17 \mathrm{~mm}$ compared to the three-axis force/torque sensor in [17] [24]. The height is about $14 \mathrm{~mm}$. Inside the mechanical structure, six optical sensors are mounted to the top and bottom using two flexible circuits (by Sunhayato Corp., Japan) as shown in Figure 2. Each sensor is

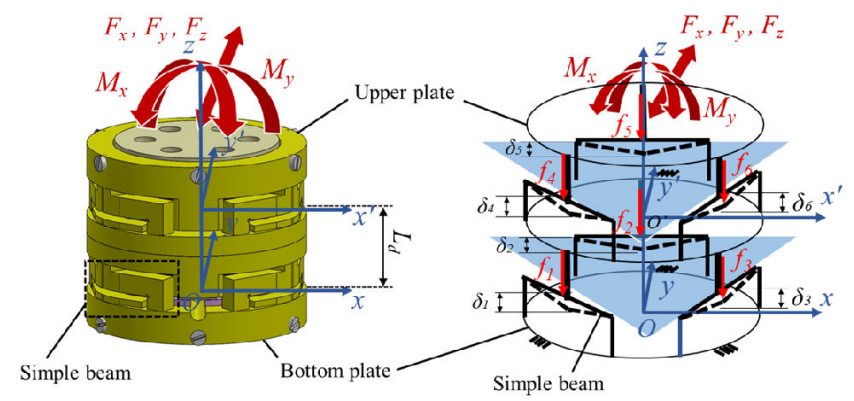

Figure 3: Sensor structure (section of simply-supported beam) dimension and design variables
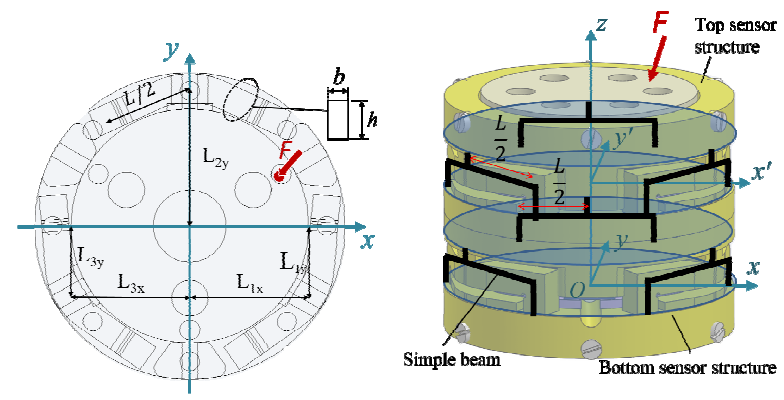

Figure 4: Sensor structure cross-section view and beam arrangement. 

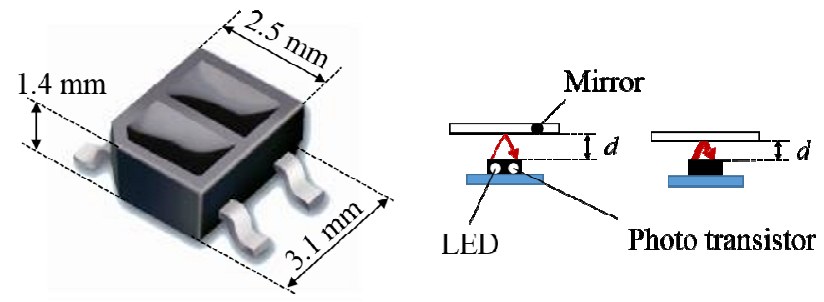

Figure 5: Assembly of the five-axis force/torque sensor: (a) Exploded view and (b) prototype of the five axis force/torque sensor.

opposed by a mirror. Here, we assembled QRE1113 reflectance sensors from Fairchild Semiconductor Corp. comprised of two parts - an IR emitting LED and an IR sensitive phototransistor (Figure 5). The LED is the light source; the reflected light is transmitted to the phototransistor via the mirror. The phototransistor converts the reflected light intensity into a voltage. The closer the distance between the mirror and the optical sensor is, the larger an amount of the reflected light intensity, and in proportion to the reflected light intensity, the output voltage of the phototransistor increases. When external forces or moments are applied on the upper plate of the sensor, the six deflections $\left(\delta_{1}, \delta_{2}, \delta_{3}, \delta_{4}\right.$, $\delta_{5}$, and $\delta_{6}$ ) are measured by the six optical sensors (Figure 3 ). Processing the amount of the six deflections, force/torque components can be calculated. In general, the optical sensor is sensitive to external light, so the housing covering the sensing structure is painted in black colour and, hence, blocks external light as shown in Figure 1.

Figure 6 (a) shows the voltage output of the optical sensor with respect to a distance variation between the sensor and opposed mirror. As the voltage output change within $0.3 \mathrm{~mm}$ (which is the maximum deflection of each simply-supported beam) is minimal, an amplifier has been designed to obtain larger voltage changes (see Figure 6 (b) blue area). All wires for measuring the voltage output of the six optical sensors and for the power supply of the amplifier are guided within a $1.2 \mathrm{~mm}$ spring wire (shield cable) as shown in Figure 1.

\section{Mathematical model of the beam}

The sensor structure is comprised of six simply-supported beams and, from their deflections $\left(\delta_{1}, \delta_{2}, \delta_{3} \delta_{4}, \delta_{5}\right.$, and $\left.\delta_{6}\right)$, the force components $\left(f_{1}, f_{2}, f_{3}, f_{4}, f_{5}\right.$, and $\left.f_{6}\right)$ can be calculated by multiplying the six deflections by spring coefficients $k$ as shown in Equations. 1 to 6.

From these six force components, $F_{z}, M_{1 x}, M_{2 x}, M_{1 y}, M_{2 y}$, $M_{x}$, and $M_{y}$ can be calculated using Equations 7 to 12 .

$$
\begin{gathered}
f_{1}=k_{1} \delta_{1} \\
f_{2}=k_{2} \delta_{2} \\
f_{3}=k_{3} \delta_{3} \\
f_{4}=k_{4} \delta_{4} \\
f_{5}=k_{5} \delta_{5} \\
f_{6}=k_{6} \delta_{6} \\
F_{z}=\left(f_{1}+f_{2}+f_{3}+f_{4}+f_{5}+f_{6}\right) / 2 \\
M_{x}=M_{2 x}-M_{1 x}, M_{y}=M_{2 y}-M_{1 y} \\
M_{1 x}=-L_{2 y} \cdot f_{5}+L_{1 y} \cdot f_{6}+L_{3 y} \cdot f_{4} \\
M_{1 y}=L_{1 x} \cdot f_{6}-L_{3 x} \cdot f_{4}
\end{gathered}
$$
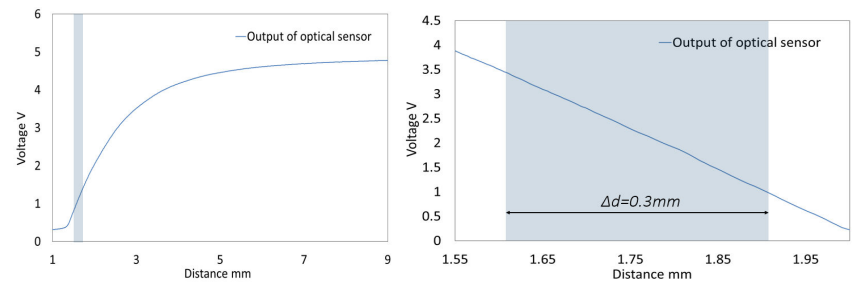

Figure 6: Characteristic curve of output voltage of optical sensor according to changing $d$ : a) without amplifier b) with inverted amplifier.

$$
\begin{aligned}
& M_{2 x}=-L_{2 y} \cdot f_{2}+L_{1 y} \cdot f_{3}+L_{3 y} \cdot f_{1} \\
& M_{2 y}=L_{1 x} \cdot f_{3}-L_{3 x} \cdot f_{1} \\
& M_{x}=L_{3 y} \cdot f_{1}-L_{2 y} \cdot f_{2}+L_{1 y} \cdot f_{3}-L_{3 y} \cdot f_{4}+L_{2 y} \cdot f_{5}-L_{1 y} \cdot f_{6} \\
& M_{y}=-L_{3 x} \cdot f_{1}+L_{1 x} \cdot f_{3}+L_{3 x} \cdot f_{4}-L_{1 x} \cdot f_{6}
\end{aligned}
$$

The force components $F_{x}$ and $F_{y}$ are calculated by the difference of the two moment components divided by $L_{d}$ using Equations 15 and 16 (see Figure 43).

$$
\begin{aligned}
& F_{x}=\frac{M_{2 y}-M_{1 y}}{L_{d}} \\
& =\frac{-L_{3 x} \cdot f_{1}+L_{1 x} \cdot f_{3}+L_{3 x} \cdot f_{4}-L_{1 x} \cdot f_{6}}{L_{d}} \\
& F_{y}=\frac{M_{2 x}-M_{1 x}}{L_{d}} \\
& =\frac{L_{3 y} \cdot f_{1}-L_{2 y} \cdot f_{2}+L_{1 y} \cdot f_{3}-L_{3 y} \cdot f_{4}+L_{2 y} \cdot f_{5}-L_{1 y} \cdot f_{6}}{L_{d}}
\end{aligned}
$$

The force/torque measurable range can be determined by the spring coefficients $k$, which can be changed by adjusting the beams' properties: length $L$, width $b$, height $h$, and material elastic coefficient $E$, as shown in Figure 4 and Equations 17 to 19 [25].

$$
\begin{aligned}
I & =\frac{b h^{3}}{12} \\
\delta & =\frac{f L^{3}}{48 E I} \\
k & =\frac{48 E I}{L^{3}}
\end{aligned}
$$

To convert the spring coefficients multiplied by six deflections to the voltages of each of the six optical sensors, the coefficients $m$ should be measured by experiment as shown in Equations 20 to 25.

$$
\begin{gathered}
f_{1}=k \delta_{1}=m_{1} v_{1} \\
f_{2}=k \delta_{2}=m_{2} v_{2} \\
f_{3}=k \delta_{3}=m_{3} v_{3} \\
f_{4}=k \delta_{4}=m_{4} v_{4} \\
f_{5}=k \delta_{5}=m_{5} v_{5} \\
f_{6}=k \delta_{6}=m_{6} v_{6}
\end{gathered}
$$

Finally, the stiffness matrix can be obtained (see Eq. 26). 


$$
\begin{gathered}
{\left[\begin{array}{c}
F_{x} \\
F_{y} \\
F_{z} \\
M_{x} \\
M_{y}
\end{array}\right]=\boldsymbol{k} \cdot \boldsymbol{v}=\left[\begin{array}{cccccc}
k_{v 11} & k_{v 12} & k_{v 13} & k_{v 14} & k_{v 15} & k_{v 16} \\
k_{v 21} & k_{v 22} & k_{v 23} & k_{v 24} & k_{v 25} & k_{v 26} \\
k_{v 31} & k_{v 32} & k_{v 33} & k_{v 34} & k_{v 35} & k_{v 36} \\
k_{v 41} & k_{v 42} & k_{v 43} & k_{v 44} & k_{v 45} & k_{v 46} \\
k_{v 51} & k_{v 52} & k_{v 53} & k_{v 54} & k_{v 55} & k_{v 56}
\end{array}\right] \cdot\left[\begin{array}{c}
v_{1} \\
v_{2} \\
v_{3} \\
v_{4} \\
v_{5} \\
v_{6}
\end{array}\right]} \\
=\left[\begin{array}{cccccc}
-m_{1} L_{3 x} \\
\frac{L_{d}}{m_{1} L_{3 y}} & \frac{-m_{2} L_{2 y}}{L_{d}} & \frac{m_{3} L_{1 y}}{L_{d}} & \frac{-m_{4} L_{3 y}}{L_{d}} & \frac{m_{5} L_{2 y}}{L_{d}} & \frac{-m_{6} L_{1 y}}{L_{d}} \\
\frac{m_{1}}{2} & \frac{m_{2}}{2} & \frac{m_{3}}{2} & \frac{m_{4}}{2} & \frac{m_{5}}{2} & \frac{m_{6}}{2} \\
m_{1} L_{3 y} & -m_{2} L_{2 y} & m_{3} L_{1 y} & -m_{4} L_{3 y} & m_{5} L_{2 y} & -m_{6} L_{1 y} \\
-m_{1} L_{3 x} & 0 & m_{3} L_{1 x} & m_{4} L_{3 x} & 0 & -m_{6} L_{1 x}
\end{array}\right] \cdot\left[\begin{array}{c}
L_{2} \\
v_{1} \\
v_{2} \\
v_{4} \\
v_{5} \\
v_{6}
\end{array}\right]
\end{gathered}
$$

\section{Sensor structure FEM simulations}

To verify the structure of our proposed sensor principle, several FEM simulations have been conducted. A force of 2.5 $\mathrm{N}$ has been exerted at different locations to the top plate of the sensor (see Figure 7). The FEM simulation shows the six beams are deformable when external force/torque components are applied. The following material properties were considered: tensile modulus of $1,283 \mathrm{MN} / \mathrm{m}^{2}$, mass density of $1,020 \mathrm{~kg} / \mathrm{m}^{3}$, tensile strength of $42,400 \mathrm{kN} / \mathrm{m}^{2}$; these assumptions are based on information provided by the manufacturer of the rapid prototyping machine.

In Figure 7 (a), the applied force is applied equally to the upper surface of the sensor, so that this results in an axial force $F_{z}$ only. Hence, the six simply-supported beams deflect equally. Exerting the force value of $2.5 \mathrm{~N}$ in negative $\mathrm{x}-$ direction at the top plate yields in the force $F_{x}$ and the moment $\mathrm{M}_{\mathrm{y}}$ (see Figure 7 (b)). The moment $\mathrm{M}_{\mathrm{y}}$ causes the three deflections on the bottom sensor structure to be larger than the ones on the top sensor. A similar case effects the sensor structure, when applying $2.5 \mathrm{~N}$ in negative y-direction. $\mathrm{F}_{\mathrm{y}}$ and $\mathrm{M}_{\mathrm{x}}$ generate a larger displacement to the simplysupported beams of the bottom sensor structure as it can be seen in Figure 7 (c). In Figure 7 (d) and (e), the force $F_{z}$ and the moment $\mathrm{M}_{\mathrm{y}}$ and $\mathrm{M}_{\mathrm{x}}$, respectively, are caused by a force of $2.5 \mathrm{~N}$ exerted to top structure in negative $\mathrm{z}$-direction, $5 \mathrm{~mm}$ away from the central axis. The FEM simulations show that the top structure is deformed in the same amount as the bottom structure.

\section{CAlibration Methodology AND Results}

Figure 8 shows the calibration device and illustrates the methodology the tool offers. Calibration is an essential step to convert the voltages of the optical sensors of the five-axis force/torque sensor to physical values (in our case, force/torque components), and find a calibration matrix which relates the output voltages the optical sensors to the physical values of the five-axis force/torque sensor. For this reason, a calibration device is presented that exerts force/torque conditions on the five-axis force/torque sensor to find the calibration matrix. The calibration device consists of a linear guide, a load fixture, a load cell (ATI Nano 17) with a fixture, and a sensor base (see Figure 8 (a)). A variety of force/torque conditions can be applied to the five-axis force/torque sensor by pulling a wire along a linear motor
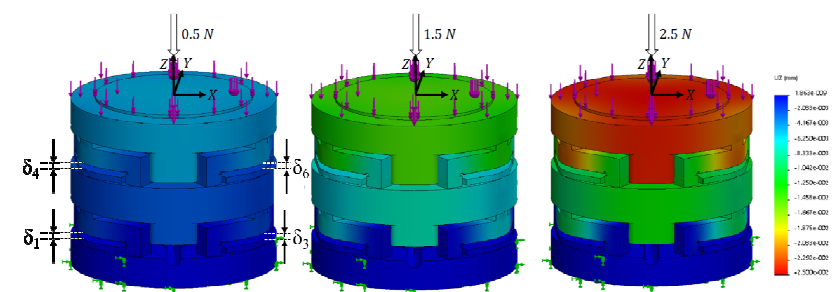

(a)
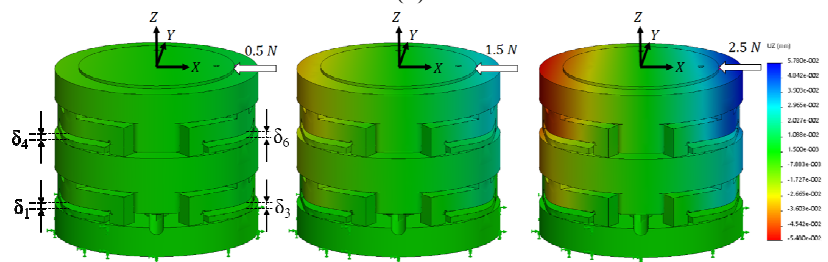

(b)
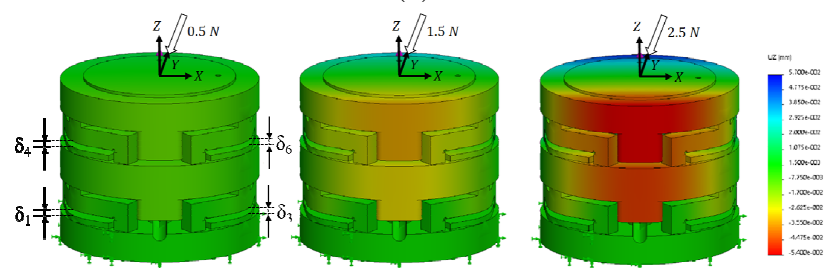

(c)
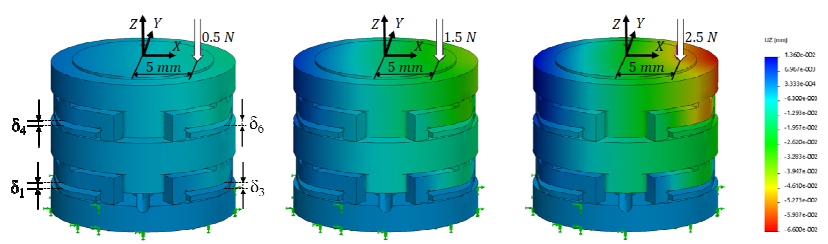

(d)
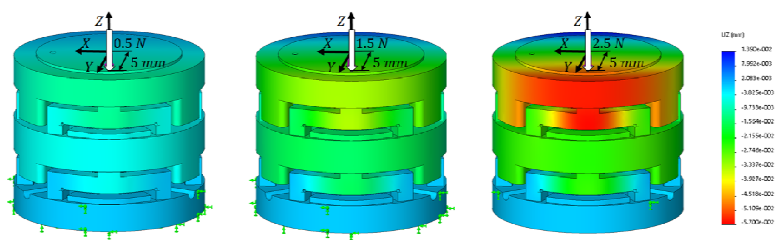

(e)

Figure 7: FEM Simulation: Applied forces and moments result in deflections of the six simply-supported beams by the six force components $\left(\mathrm{f}_{1}, \mathrm{f}_{2}, \mathrm{f}_{3}, \mathrm{f}_{4}, \mathrm{f}_{5}\right.$, and $\left.\mathrm{f}_{6}\right)$.

system. Due to Newton's third law, the pulling forces are transmitted via the wire to the sensor (even if the wire might be extensible). At the same time, the output voltages of the five-axis force/torque sensor were recorded by an $\mathrm{A} / \mathrm{D}$ converter (Analog-to-Digital converter). The calibration device can apply an amount of external force/torque components, such as a combination of $F_{x}$ and $M_{y}$, or $F_{y}$ and $M_{x}$ as shown in Figure 8 (b). In addition, the calibration device can apply an amount of external force/torque components $F_{z}$, or a combination of $F_{z}$ and $M_{x}$, or $F_{z}$ and $M_{y}$. All characteristic curves were obtained while loading the force/torque conditions (Figure 8 (a)).

The experiment results show all characteristic curves between the output voltages of the five-axis force/torque sensor and the five-axis force/torque sensor regarding various force/torque conditions as shown in Figure 9. Regarding the force/torque conditions, all data sets (between 

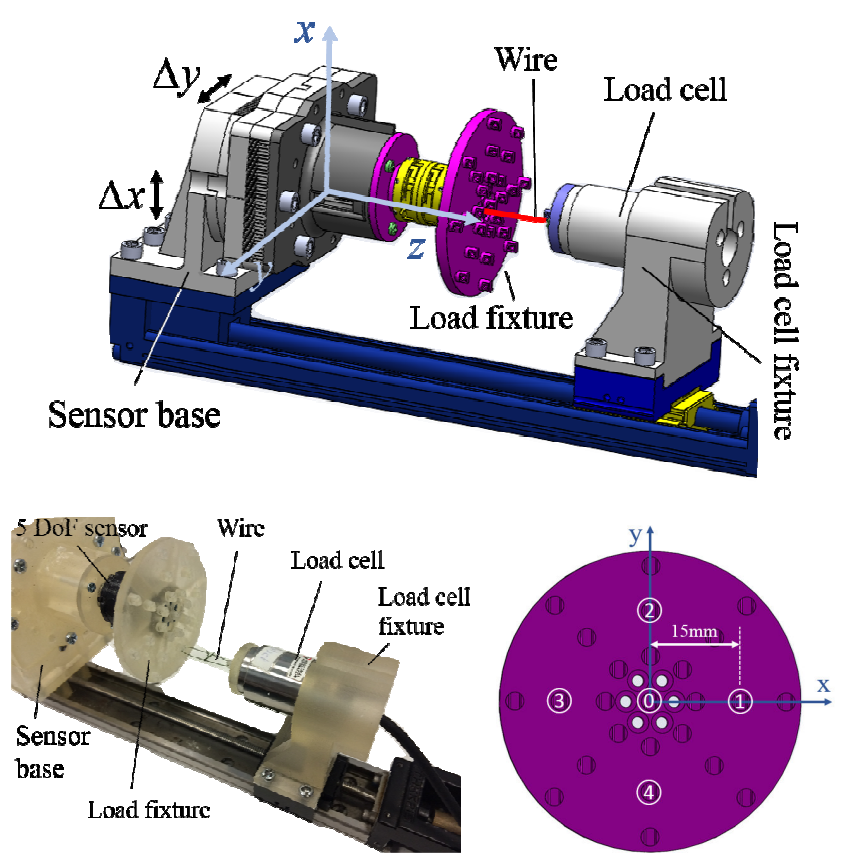

(a)
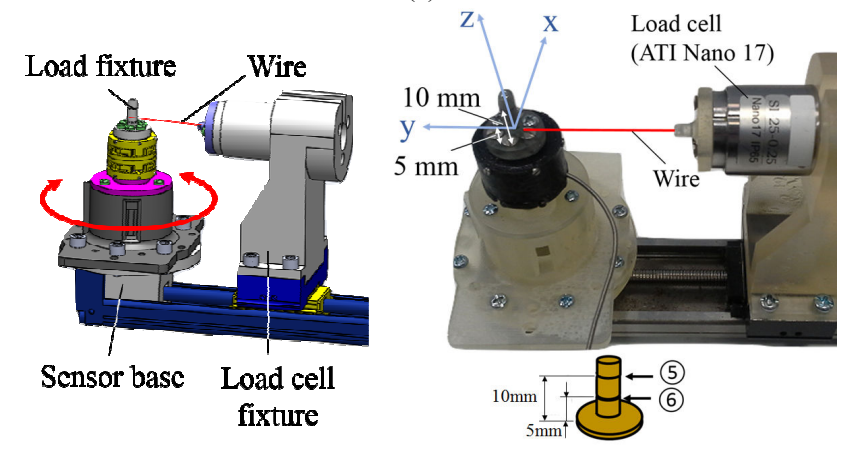

(b)

Figure 8: Sensor calibration device: A number of force/torque conditions can be applied using a wire to exert tensile force. (a) Various force/torque conditions $\left(\mathrm{F}_{\mathrm{z}}, \mathrm{M}_{\mathrm{x}}\right)$ (2) (4), $\left(\mathrm{F}_{\mathrm{z}}, \mathrm{M}_{\mathrm{y}}\right)$ (1)(3), or $\mathrm{F}_{\mathrm{z}}(0)$ are applied on the load fixture; (b) Force/torque conditions $\left(\mathrm{F}_{\mathrm{x}}, \mathrm{M}_{\mathrm{y}}\right)$ or $\left(\mathrm{F}_{\mathrm{y}}, \mathrm{M}_{\mathrm{x}}\right)$ are applied on the load fixture. The sensor base can be rotated, and fixed by the screws to apply various positive and negative force/torque conditions on the two points (5) (6) of the load fixture are applied.

the output voltages of the five-axis force/torque sensor and physical force/torque components) were acquired.

This calibration data set was used to generate a $5 \times 6$ calibration or stiffness matrix, which converts the six output voltages to five physical values of force and torque. Therefore the matrix can be later on multiplied by any sixelement voltage vector (column) (see Equation 25) to obtain the sensor output.

Multiple Linear Regression (MLR) finds the relationship between two or more independent variables and a dependent variable by fitting a linear equation to the observed data [26]. In this implementation, every value of an independent variable, namely each value of the output voltage of the photo sensors of the five-axis force/torque sensor, is associated with a value of the dependent variables, i.e., the force and torque components $F_{x}, F_{y}, F_{z}, M_{x}$, and $M_{y}$. By applying MLR, the decoupling stiffness matrix (calibration matrix) is calculated as shown in Equations 26.

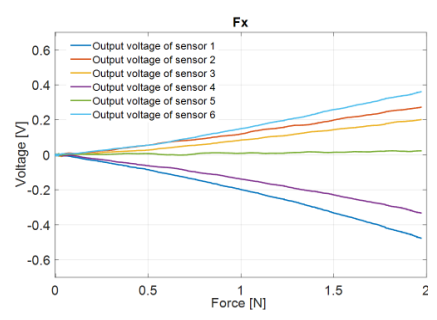

(a)

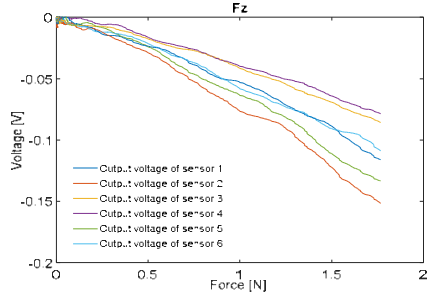

(c)

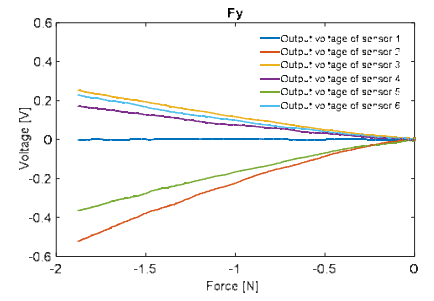

(b)

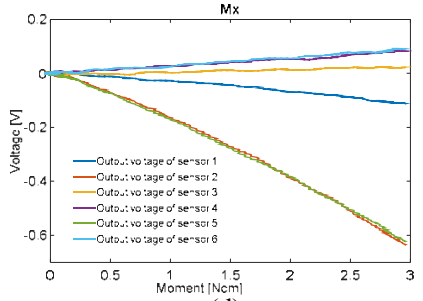

(d)

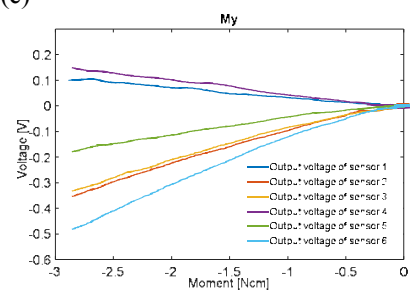

(e)

Figure 9: Examples of characteristic curve between physical force/torque components and the output voltage of the five-axis force/torque sensor: (a) $\left(+\mathrm{F}_{\mathrm{x}},+\mathrm{M}_{\mathrm{y}}\right)$ applying force/torque conditions on the load fixture (5), (b) ($\mathrm{F}_{\mathrm{y}},+\mathrm{M}_{\mathrm{x}}$ ) applying force/torque conditions on the load fixture (5), (c) $+\mathrm{F}_{\mathrm{z}}$ applying force condition on the load fixture (0), (d) $\left(+\mathrm{F}_{\mathrm{z}},+\mathrm{M}_{\mathrm{x}}\right)$ applying force/torque conditions on the load fixture (2), (e) $\left(+\mathrm{F}_{\mathrm{z}},-\mathrm{M}_{\mathrm{y}}\right)$ applying force/torque conditions on the load fixture (1).

$$
\begin{aligned}
& =\left[\begin{array}{cccccc}
k_{v 11} & k_{v 12} & k_{v 13} & k_{v 14} & k_{v 15} & k_{v 16} \\
k_{v 21} & k_{v 22} & k_{v 23} & k_{v 24} & k_{v 25} & k_{v 26} \\
k_{v 31} & k_{v 32} & k_{v 33} & k_{v 34} & k_{v 35} & k_{v 36} \\
k_{v 41} & k_{v 42} & k_{v 43} & k_{v 44} & k_{v 45} & k_{v 46} \\
k_{v 51} & k_{v 52} & k_{v 53} & k_{v 54} & k_{v 55} & k_{v 56}
\end{array}\right] \times\left[\begin{array}{c}
v_{1} \\
v_{2} \\
v_{3} \\
v_{4} \\
v_{5} \\
v_{6}
\end{array}\right]=\left[\begin{array}{c}
F_{x} \\
F_{y} \\
F_{z} \\
M_{x} \\
M_{y}
\end{array}\right] \\
& k=\left[\begin{array}{ccccccc}
-11.0577 & 4.3205 & 1.6458 & 10.7029 & -1.3227 & -2.8698 \\
5.4595 & 8.5276 & -4.7401 & -2.9240 & -9.8544 & 0.9312 \\
-0.4006 & -0.1695 & 1.0580 & -4.8603 & -4.5166 & -4.9457 \\
0.7012 & 2.3349 & -2.4202 & 3.2485 & -6.6556 & 3.7957 \\
6.1295 & -1.1886 & 1.4440 & -11.6688 & -0.7868 & 3.8449
\end{array}\right]
\end{aligned}
$$

\section{VERIFICATION AND EVALUATION}

To verify the calibration matrix, a set of the experiments were carried out using the calibration device. Various force/torque conditions (while loading the force/torque conditions) were applied on the five-axis force/torque sensor to compare real force/moment components with measured force/torque by our new five-axis force/torque sensor calculated by the calibration matrix.

Evaluating the sensor's accuracy, the errors of $F_{x}, F_{y}, F_{z}$ $M_{x}$ and $M_{y}$ components have been calculated with the maximum measured forces and moments (see Table I). With respect to crosstalk, all force/torque components except $F_{z}$ 
are less than $6.7 \%$, with $F_{z}$ being less than $8.2 \%$ as shown in Table II.

\section{CONCLUSIONS AND FUTURE WORKS}

In this paper, a new five-axis force/torque sensor based on the optoelectronic sensing principle has been presented. The sensor is made of two three-axis force/torque sensors mounted in series. Reflecting on the set design input requirements, we achieved to create a multi-axis sensor with a diameter of $17 \mathrm{~mm}$ and a height of $14 \mathrm{~mm}$ - these are comparable with dimensions of commercially available force/torque sensors such as the ATI Nano 17 sensor. Due to the chosen fabrication method and integrated electronic components, our sensor is clearly low-cost. The simplysupported beam structure is simple and, hence, allows effortless adaptation when embedding into robotic manipulators. The force/torque sensor is able to measure forces of up to $2 \mathrm{~N}$ and moments of up to $3 \mathrm{Ncm}$. These ranges can be varied by choosing different materials for the sensor structure and, hence, by changing the stiffness coefficient of the simply-supported beams.

TABLE I. SENSOR PERFORMANCE PROPERTY: RANGE AND MAXIMUM ERROR

\begin{tabular}{c|c|c}
$\begin{array}{c}\text { Force / } \\
\text { moment }\end{array}$ & Range & Maximum error \\
\hline$F_{x}$ & $+/-2.0 \mathrm{~N}$ & $12.4 \%$ \\
\hline$F_{y}$ & $+/-2.0 \mathrm{~N}$ & $26.7 \%$ \\
\hline$F_{z}$ & $+/-2.0 \mathrm{~N}$ & $13.1 \%$ \\
\hline$M_{x}$ & $+/-3.0 \mathrm{Ncm}$ & $6.1 \%$ \\
\hline$M_{y}$ & $+/-3.0 \mathrm{Ncm}$ & $11.3 \%$
\end{tabular}

TABLE II. SENSOR PERFORMANCE PROPERTY: CROSSTALK

\begin{tabular}{c|r|r|r|r|r}
$\begin{array}{c}\text { Force } / \\
\text { moment }\end{array}$ & \multicolumn{1}{|c|}{$\boldsymbol{F}_{z}$} & \multicolumn{1}{|c|}{$\boldsymbol{F}_{\boldsymbol{x}} \boldsymbol{M}_{\boldsymbol{y}}$} & $\boldsymbol{F}_{\boldsymbol{y}} \boldsymbol{M}_{\boldsymbol{x}}$ & \multicolumn{1}{|c|}{$\boldsymbol{F}_{z} \boldsymbol{M}_{\boldsymbol{x}}$} & \multicolumn{1}{c}{$\boldsymbol{F}_{z} \boldsymbol{M}_{\boldsymbol{y}}$} \\
\hline$F_{x}$ & $6.7 \%$ & - & $2.8 \%$ & $2.3 \%$ & $2.5 \%$ \\
\hline$F_{y}$ & $0.9 \%$ & $6.0 \%$ & - & $5.0 \%$ & $1.5 \%$ \\
\hline$F_{z}$ & - & $8.2 \%$ & $15.7 \%$ & - & - \\
\hline$M_{x}$ & $0.9 \%$ & $0.8 \%$ & - & - & $4.8 \%$ \\
\hline$M_{y}$ & $1.2 \%$ & - & $8.7 \%$ & $4.2 \%$ & -
\end{tabular}

\section{REFERENCES}

[1] P. Neto, J.N. Pires, A.P. Moreira, "High-level programming and control for industrial robotics: using a hand-held accelerometer-based input device for gesture and posture recognition", Industrial Robot: An International Journal, Vol. 37(2), pp. 137 - 147, 2010.

[2] Y. Qing-Xiao, Y. Can, F. Zhuang, Z. Yan-Zheng, "Research of the Localization of Restaurant Service Robot", International Journal of Advanced Robotic Systems, Vol. 7(3), pp. 227-238, 2010.

[3] K.P. Hawkins, P.M. Grice, T.L. Chen, C.-H. King, C.C. Kemp, "Assistive Mobile Manipulation for Self-Care Tasks Around the Head", IEEE Symposium on Computational Intelligence in Robotic Rehabilitation and Assistive Technologies, 2014

[4] A.T. Hillel, A. Kapoor, N. Simaan, R.H. Taylor, P. Flint, "Applications of Robotics for Laryngeal Surgery," Laryngeal Cancer Otolaryngologic Clinics of North America, Nasir Bhatti \& Ralph P. Tufano Eds., Vol. 41(4), pp. 781-791, 2008.

[5] H. Miwa, K. Itoh, M. Matsumoto, M. Zecca, H. Takanobu, S. Roccella, M.C. Carrozza, P. Dario, A. Takanishi, "Effective Emotional Expressions with Emotion Expression Humanoid Robot WE-4RII - Integration of Humanoid Robot Hand RCH-1", IEEE/RSJ International Conference on Intelligent Robots and Systems, pp. 2203 $-2208,2011$.

[6] C. Breazeal, "Function Meets Style: Insights from Emotion Theory Applied to HRI", IEEE Transactions in Systems, Man, and Cybernetics, Part C, 34(2), pp. 187-194, 2003.
[7] H. Liu, K.C. Nguyen, V. Perdereau, J. Bimbo, J. Back, M. Godden, L.D. Seneviratne, K. Althoefer, "Finger contact sensing and the application in dexterous hand manipulation", Autonomous Robots Vol. 39(1), pp. 25-41, 2015.

[8] J. Bimbo, P. Kormushev, K. Althoefer, H. Liu, "Global estimation of an object's pose using tactile sensing", Advanced Robotics, Special Issue: Humanoid Robotics (1), Vol. 29(5), 2015.

[9] R. Fukuia, M. Watanabeb, M. Shimosaka, T. Sato, "Hand shape classification in various pronation angles using a wearable wrist contour sensor", Advanced Robotics, Vol. 29(1), 2015.

[10] H. Hasegawa, Y. Suzuki, A. Ming, M. Ishikawa, M. Shimojo, "Robot Hand Whose Fingertip Covered with Net-Shape Proximity Sensor Moving Object Tracking Using Proximity Sensing“, Journal of Robotics and Mechatronics Vol.23(3), pp. 328-337, 2011.

[11] T. Mukai, S. Hirano, M. Yoshida, H. Nakashima, S. Guo, Y. Hayakawa, "Tactile-Based Motion Adjustment for the Nursing-Care Assistant Robot RIBA", IEEE International Conference on Robotics and Automation, pp. 5435- 5441, 2011.

[12] C.-H. King, T.L. Chen, A. Jain, C.C. Kemp," Towards an Assistive Robot that Autonomously Performs Bed Baths for Patient Hygiene", IEEE/RSJ International Conference on Intelligent Robots and Systems, pp. 319 - 324, 2010.

[13] Y. Yamada, S. Hayakawa, N. Tsuchida, "Shaft Inserting Task into Inclined Hole of Moving Object by Using One Dimensional PSDs Sensor", IEEE International Workshop on Advanced Motion Control, pp. 659-664, 2004.

[14] D.V. Dao, T. Toriyama, J. Wells, S. Sugiyama, "Silicon Piezoresistive Six-Degree of Freedom Micro Force-Moment Sensor", IEEE International Conference on Micro Electro Mechanical Systems, pp. $312-315,2002$.

[15] U. Seibold, B. Kubler, G. Hirzinger, "Prototype of Instrument for Minimally Invasive Surgery with 6-Axis Force Sensing Capability", IEEE International Conference on Robotics and Automation Barcelona, pp.496-501, 2004.

[16] Y. Jia, X. Chen, Q. Ni, L. Li, C. Ju, "Dependence of the Impact Response of Polyvinylidene Fluoride Sensors on Their Supporting Materials' Elasticity", Sensors, pp. 8669-8678, 2013.

[17] Y. Noh, S. Sareh, J. Back, H.A. Wurdemann, T. Ranzani, E.L. Secco, A Faragasso, H. Liu, K. Althoefer, "A three-axial body force sensor for flexible manipulators", IEEE International Conference on Robotics and Automation, pp. 6388-6369.

[18] C.J. Pacheco, A.C. Bruno, "A Noncontact Force Sensor Based on a Fibre Bragg Grating and Its Application for Corrosion Measurement", Sensors, pp.11476-11489, 2013.

[19] Y. Noh, K. Nagahiro, Y. Ogura, H. Ishii, J. Solis, K. Hatake, A. Takanishi, "Development of the airway management training system WKA-1 designed to embed arrays of sensors into a conventional mannequin", IEEE/RSJ International Conference on Intelligent Robots and Systems, pp. 1296-1301, 2007.

[20] D. Tsetserukou, R. Tadakuma, H. Kajimoto, and S. Tachi, "Optical torque sensors for implementation of local impedance control of the arm of humanoid robot," IEEE Int. Conf. on Robotics and Automation, pp. 1674-1679, 2006.

[21] Y. Noh, A. Shimomura, M. Segawa, H. Ishii, J. Solis, A. Takanishi, K. Hatake, "Development of Tension/Compression Detection Sensor System designed to acquire quantitative force information while training the airway management task", IEEE/ASME Int. Conf. on Advanced Intelligent Mechatronics, pp. 1264-1269, 2009.

[22] G. Pallia, S. Pirozzib, "A miniaturized optical force sensor for tendondriven mechatronic systems: Design and experimental evaluation", Mechatronics, Vol 22(8), pp. 1097-1111, 2012.

[23] J.-C. Kim, K.-S. Kim, S. Kim, "Note: A compact three-axis optical force/torque sensor using photo-interrupters", Review of Scientific Instruments 84, 126109 (2013).

[24] Y. Noh, E.L. Secco, S. Sareh, H.A. Wurdemann, A. Faragasso, J. Back, H. Liu, E. Sklar, K. Althoefer, "A continuum body force sensor designed for flexible surgical robotics devices", Annual International Conference of the IEEE Engineering in Medicine and Biology Society, pp. 3711-3714, 2014.

[25] F. Beer, Jr., E. Russell Johnston, Mechanics of Materials, Paperback (McGraw-Hill), 4th edition.

[26] L.L. Nathans, F.L. Oswald, K. Nimon, "Interpreting Multiple Linear Regression: A Guidebook of Variable Importance", Practical Assessment, Research \& Evaluation, Vol. 17(9), pp.1-19, 2012. 